\title{
On the Minimum Cable Tensions for the Cable-Based Parallel Robots
}

\author{
Peng Liu, ${ }^{1}$ Yuanying Qiu, ${ }^{1}$ Yu Su, ${ }^{2}$ and Jiantao Chang ${ }^{1}$ \\ ${ }^{1}$ Key Laboratory of Ministry of Education for Electronic Equipment Structure Design, Xidian University, Xian 710071, China \\ ${ }^{2}$ School of Mechanical and Electronic Engineering, Xidian University, Xian 710071, China
}

Correspondence should be addressed to Peng Liu; 200304405liupeng@163.com

Received 19 May 2014; Accepted 15 July 2014; Published 4 August 2014

Academic Editor: Antonio Bandera

Copyright (C) 2014 Peng Liu et al. This is an open access article distributed under the Creative Commons Attribution License, which permits unrestricted use, distribution, and reproduction in any medium, provided the original work is properly cited.

\begin{abstract}
This paper investigates the minimum cable tension distributions in the workspace for cable-based parallel robots to find out more information on the stability. First, the kinematic model of a cable-based parallel robot is derived based on the wrench matrix. Then, a noniterative polynomial-based optimization algorithm with the proper optimal objective function is presented based on the convex optimization theory, in which the minimum cable tension at any pose is determined. Additionally, three performance indices are proposed to show the distributions of the minimum cable tensions in a specified region of the workspace. An important thing is that the three performance indices can be used to evaluate the stability of the cable-based parallel robots. Furthermore, a new workspace, the Specified Minimum Cable Tension Workspace (SMCTW), is introduced, within which all the minimum tensions exceed a specified value, therefore meeting the specified stability requirement. Finally, a camera robot parallel driven by four cables for aerial panoramic photographing is selected to illustrate the distributions of the minimum cable tensions in the workspace and the relationship between the three performance indices and the stability.
\end{abstract}

\section{Introduction}

Cable-based parallel robots whose end-effectors are manipulated by motors that can extend or retract the cables are a type of robotic manipulator that has recently attracted interest for large workspace manipulation tasks. They show several promising advantages over their rigid-link counterparts, such as simple light-weight mechanical structure, low moment inertia, large reachable workspace, and high-speed motion. Thus, a wide variety of cable-based robots have been used in medical rehabilitation, material transportation, wind tunnel experiment, astronomical observation, and other fields [14]. However, cable-based parallel robots do sacrifice some accuracy and stability due to using the cables instead of links. Based on the number of cables $(m)$ and the number of degrees of freedom $(n)$, cable-based parallel robots are classified into three categories, that is, the incompletely restrained positioning mechanisms $(m<n+1)$, the completely restrained positioning mechanisms $(m=n+1)$, and the redundantly restrained positioning mechanisms $(m>n+1)$ [5]. The different methods are employed to determine cable tensions in the above-mentioned three cable-based parallel robots, in which the determination of cable tensions is based on the convex optimization algorithm for the completely restrained and redundantly restrained cable-based parallel robots [6]. Some researchers have worked on the determination of the cable tensions in cable-based parallel robots. Hassan and Khajepour [7] presented a method based on convex theory for the tensions. However, the cable tensions were solved by using Dykstra's projection algorithm, which is an iterative algorithm that is time consuming. Mikelsons et al. [8] developed a noniterative algorithm for the safe tensions and proved its continuity. Nevertheless, the algorithm suffers from poorexecution time. Borgstrom et al. [9] adopted the minimum2 norm as the optimal objective function to solve tensions and suggested the minimum- 2 norm is good at continuity as compared with the minimum-1 norm. But the cable tensions are prone to the small forces of the zonotope in the space of the platform wrenches. Gosselin and Grenier [6] presented a noniterative algorithm of cable tension determining and proposed four optimal objective functions for the algorithm. Indeed, to the best of our knowledge, all of the above 
literatures do not consider the distribution of the minimum cable tension in the whole workspace and combine minimum cable tensions and workspace to investigative the stability of cable-based parallel robots.

This paper addresses the minimum cable tension distributions in the workspace for cable-based parallel robots. First of all, the determination of the cable tensions of completely restrained parallel robots is presented. This problem is treated as a noniterative polynomial-based optimization algorithm with an optimal objective function based on the convex optimization theory. Moreover, the minimum cable tension at any pose is determined and SMCTW is introduced based on the concepts of minimum cable tension and workspace. Subsequently, three performance indices are developed to explain the distributions of the minimum cable tensions in the workspace. Additionally, the results and discussion are given in Section 2. Finally, a camera robot for aerial panoramic photographing is selected as an example to illustrate the three performance indices above and the relationship between the three performance indices and the stability. It is pointed out that the three performance indices can be employed to evaluate the stability of a cable-based parallel robot.

\section{Modeling of Cable-Based Parallel Robots}

2.1. Notation and Wrench Matrix. A cable-based parallel robot is represented schematically in Figure 1. It consists of a moving rigid platform connected to the base by a set of $m$ cables attached to the platform and to fixed pulleys on which the cables are wound. The position and orientation of the platform can be controlled by controlling length of their respective cables.

Referring to Figure 1, a fixed reference frame, noted $(O, X, Y, Z)$, is attached to the base of the cable-based robot and is referred to as the base frame. A moving reference frame, noted $\left(O^{\prime}, X^{\prime}, Y^{\prime}, Z^{\prime}\right)$, is attached to the mobile platform, where $O^{\prime}$ is the reference point to be positioned at the robot. The orientation of the moving frame with respect to the base frame, represented by the rotation matrix $\mathbf{R}$, describes the orientation of the mobile platform with respect to the base of the mechanism. Point $A_{i}$, at which the $i$ th cable $(i=$ $1,2, \ldots, m)$ enters its spool, is assumed to be fixed relative to the base. Furthermore, the $i$ th cable is attached at point $B_{i}$ on the mobile platform and this attachment point is assumed to be fixed relative to the mobile platform. The $i$ th cable then connects points $A_{i}$ and $B_{i}$ that is assumed to be straight, its length being denoted by $L_{i}$. The contact points $A_{i}$ and $B_{i}$ are modeled as spherical joints. Then, vectors $\mathbf{a}_{i}$ and $\mathbf{b}_{i}$ are defined as the vectors connecting point $O$ to point $A_{i}$ and point $O^{\prime}$ to point $B_{i}$, respectively, expressed in the base frame. The position vector $\mathbf{P}=[x, y, z]^{T}$ of the mobile platform is given by the vector connecting point $O$ to point $O^{\prime}$. When expressed in the base frame, the unit vector along cable $i$ is noted $\mathbf{d}_{i}$ and can be written as

$$
\begin{gathered}
\mathbf{d}_{i}=\frac{\mathbf{a}_{i}-\mathbf{R} \mathbf{b}_{i}-\mathbf{P}}{L_{i}}, \quad i=1,2, \ldots, m, \\
L_{i}=\left\|\mathbf{a}_{i}-\mathbf{R} \mathbf{b}_{i}-\mathbf{P}\right\|_{2},
\end{gathered}
$$

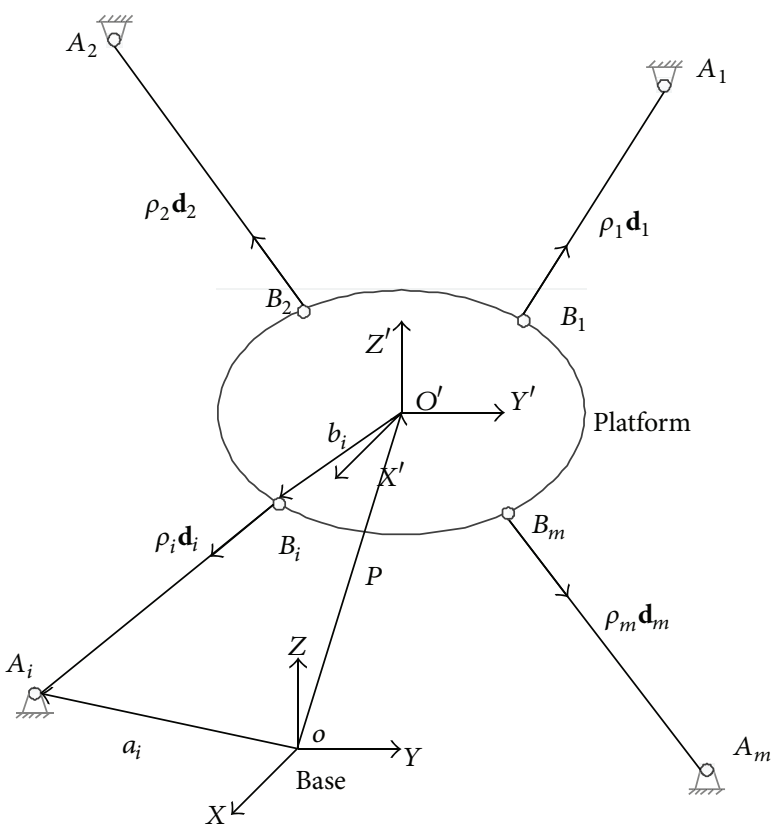

FIGURE 1: Kinematic modeling of a cable-driven parallel mechanism.

where $\|\cdot\|_{2}$ stands for the Euclidean norm of its vector argument.

When tensions are maintained in all cables, cable $i$ exerts a pure force at point $B_{i}$ on the mobile platform. The tension can be written as $t_{i} \mathbf{d}_{i}$, where $t_{i}$ is the tension in the $i$ th cable. By definition, $t_{i}$ is always nonnegative. This pure force generates a moment $\mathbf{R} \mathbf{b}_{i} \times t_{i} \mathbf{d}_{i}$ at the reference point $O^{\prime}$ of the mobile platform and the wrench (force/moment pair) applied at $O^{\prime}$ by the $i$ th cable is therefore $t_{i} \mathbf{w}_{i}$, and the wrench $\mathbf{w}_{i}$ is defined as

$$
\mathbf{w}_{i}=\left[\begin{array}{c}
\mathbf{d}_{i} \\
\mathbf{R b}_{i} \times \mathbf{d}_{i}
\end{array}\right] .
$$

Let $\mathbf{w}_{p}$ denote the wrench applied at $O^{\prime}$ by all $m$ cables of the robot. Since $\mathbf{w}_{p}$ is the sum of the cable wrenches $t_{i} \mathbf{w}_{i}$, the relationship between the tensions $t_{i}$ in the cables and the wrench $\mathbf{w}_{p}$ can be written in matrix form as [6]

$$
\mathbf{W} \mathbf{T}=\mathbf{w}_{p},
$$

where $\mathbf{T}=\left[\begin{array}{llll}t_{1} & t_{2} & \cdots & t_{m}\end{array}\right] \in \mathbb{R}^{m}$ is the vector of cable tensions and $\mathbf{W}=\left[\begin{array}{llll}\mathbf{w}_{1} & \mathbf{w}_{2} & \cdots & \mathbf{w}_{m}\end{array}\right] \in \mathbb{R}^{n \times m}$ is the pose dependent wrench matrix.

2.2. Kinematic Modeling of Cable-Based Parallel Robots. Thus using Newton method from (3), static equilibrium of cablebased parallel robots could be expressed as

$$
\mathbf{W} \mathbf{T}+\mathbf{F}_{e}+\mathbf{W}_{g}=\mathbf{0},
$$

where $\mathbf{F}_{e}=\left[\begin{array}{ll}\mathbf{F}_{R} & \mathbf{M}_{R}\end{array}\right] \in \mathbb{R}^{n}$ is the external wrench applied on the platform, where $\mathbf{F}_{R}, \mathbf{M}_{R}$ are the external force and 
moment, respectively; $\mathbf{W}_{g} \in \mathbb{R}^{n}$ is the gravitational force of the platform:

$$
\mathbf{T}_{\min }^{s} \leq \mathbf{T} \leq \mathbf{T}_{\max }^{\mathcal{s}}
$$

where the lower bound of the cable tension $\mathbf{T}_{\text {min }}^{\mathcal{S}}=$ $\left[\begin{array}{llll}t_{1, \text { min }} & t_{2, \text { min }} \cdots & t_{m, \text { min }}\end{array}\right]^{T} \in \mathbb{R}^{m}$ is required to keep cables taut; the upper bound of the cable tension $\mathrm{T}_{\max }^{\mathcal{S}}=$ $\left[\begin{array}{llll}t_{1, \max } & t_{2, \max } \cdots & t_{m, \max }\end{array}\right]^{T} \in \mathbb{R}^{m}$ is limited by the output torques of the servo motors and the breaking forces of the cables.

From (3), the cable tension solution for completely restrained parallel robots can be expressed as

$$
\mathbf{T}=\mathbf{W}^{+}\left(-\mathbf{F}_{e}-\mathbf{W}_{g}\right)+\operatorname{Null}(\mathbf{W}) \lambda
$$

where $\mathbf{W}^{+}\left(-\mathbf{F}_{e}-\mathbf{W}_{g}\right) \in \mathbb{R}^{m \times 1}$ is the special solution to the cable tensions, balancing the external loads exerted on the platform. $\mathbf{W}^{+}=\mathbf{W}^{T}\left(\mathbf{W} \mathbf{W}^{T}\right)^{-1} \in \mathbb{R}^{m \times n}$ is a MoorePenrose generalized inverse matrix of the structural matrix $\mathbf{W} . \operatorname{Null}(\mathbf{W}) \lambda$ is the homogeneous solution to the cable tensions, only changing the internal tension distribution. $\operatorname{Null}(\mathbf{W}) \in \mathbb{R}^{m \times 1}$ is the null space of the structural matrix W. $\lambda$ is an arbitrary scalar. On substituting (6) into (5), it can be acquired that

$$
\underline{\lambda} \leq \lambda \leq \bar{\lambda}
$$

where $\underline{\lambda}=\max _{1 \leq i \leq m}\left(t_{i, \min }+\mathbf{W}^{+}\left(\mathbf{F}_{e}+\mathbf{W}_{g}\right)_{i}\right) / \operatorname{Null}(\mathbf{W})_{i}$ is the lower bound of $\lambda ; \bar{\lambda}=\min _{1 \leq i \leq m}\left(t_{i, \max }+\mathbf{W}^{+}\left(\mathbf{F}_{e}+\mathbf{W}_{g}\right)_{i}\right) /$ $\operatorname{Null}(\mathbf{W})_{i}$ is the upper bound of $\lambda$.

The feasible solution $\lambda^{c}$ for $\lambda$ is a convex combination of $\bar{\lambda}$ and $\underline{\lambda}$. With (6), the feasible solution $\mathbf{T}^{c}$ is

$$
\mathbf{T}^{c}=\mathbf{W}^{+}\left(-\mathbf{F}_{e}-\mathbf{W}_{g}\right)+\operatorname{Null}(\mathbf{W}) \lambda^{c}
$$

\section{The Three Performance Indices and SMCTW Generation Algorithm}

3.1. The Cable Tension Determining of the Cable-Based Parallel Robots. Form (6), the cable tensions may present infinitely many solutions because the number of cables is larger than the degrees of freedom of the platform. Therefore, in order to obtain a unique solution, a performance index is usually optimized while using (4) and (5) as constraints. Mathematically, the determination of the cable tensions in the cables of the robot can be formulated as follows [6]:

$$
\begin{array}{ll}
\min _{T} & \boldsymbol{\beta}, \\
\text { Subject to } & \mathbf{W} \mathbf{T}+\mathbf{F}_{e}+\mathbf{W}_{g}=\mathbf{0}, \\
& \mathbf{T}_{\min }^{s} \leq \mathbf{T} \leq \mathbf{T}_{\max }^{s},
\end{array}
$$

where $\beta$ is the optimization performance index. Depending on the nature of the optimization performance index used, the above problem corresponds to different mathematical programs having different physical interpretations. In this paper, in order to control the robot steadily, the minimum variance that has a clearer physical meaning is used as an optimization performance index because all cable tensions have the least differences using it. The determination of the cable tensions in the cables of the robot with the minimum variance can be formulated as follows:

$$
\begin{array}{cl}
\boldsymbol{\beta}(\lambda)=\min & \left(\frac{1}{m}\left[\sum_{i=1}^{m}\left(t_{i}-E(\mathbf{T})\right)^{2}\right]\right) \\
\text { subject to } & \mathbf{W T}+\mathbf{F}_{e}+\mathbf{W}_{g}=\mathbf{0}, \\
& \underline{\lambda} \leq \lambda \leq \bar{\lambda},
\end{array}
$$

where $E(\mathbf{T})=\left(t_{1}+t_{2}+\cdots+t_{m}\right) / m$ is the arithmetic mean value of T. $\boldsymbol{\beta}(\lambda)$ is expressed in the form of a secondorder polynomial for the variable $\lambda$. According to the convex optimal theory, $\boldsymbol{\beta}(\lambda)$ that is a continuous and differentiable function in $[\underline{\lambda}, \bar{\lambda}]$ is obtained; thus, cable tensions are determined uniquely.

The minimum cable tension $T_{\min }$ at any position can be obtained when cable tensions are determined uniquely. From (9), the minimum cable tension $T_{\min }$ can be expressed as

$$
T_{\min }=\min (\mathbf{T})
$$

where $\min (\cdot)$ is the smallest element in a vector $\mathbf{T}$.

3.2. Three Performance Indices of Minimum Tension Distributions. In order to get any more information about the stability of a cable-based parallel robot, three performance indices are proposed to show the distributions of the minimum cable tensions in the some regions of the workspace based on the minimum cable tensions. The three performance indices are $\mathfrak{R}_{\perp}, \mathfrak{R}_{-}$, and $\mathfrak{R}$. $\mathfrak{R}_{\perp}$ and $\mathfrak{R}_{-}$represent the distributions of the minimum cable tensions on the vertical midline and on the planes paralleling with the horizontal plane, respectively. And furthermore, $\mathfrak{R}$ is proposed to describe the comprehensive distributions of the minimum cable tensions in the whole workspace. In order to make the physical meaning of the three performance indices clear, the schematic diagram relating to the three performance indices is shown in Figure 2. Without loss of generality, the optional position in the workspace is denoted by $P$; the vertical midline of the workspace is denoted by $a$; the horizontal plane on which the specified position is located is highlighted with green; the intersection of the vertical midline and the horizontal plane is denoted by $Q$; and the top position of the vertical midline is denoted by $M$.

The three performance indices can be expressed as

$$
\begin{gathered}
\mathfrak{R}_{\perp}=\frac{T_{\min }^{\$}}{T_{\min }^{+}}, \\
\mathfrak{R}_{-}=\frac{T_{\min }^{-}}{T_{\min }^{\$}} \\
\mathfrak{R}=\mathfrak{R}_{-} \times \mathfrak{R}_{\perp},
\end{gathered}
$$




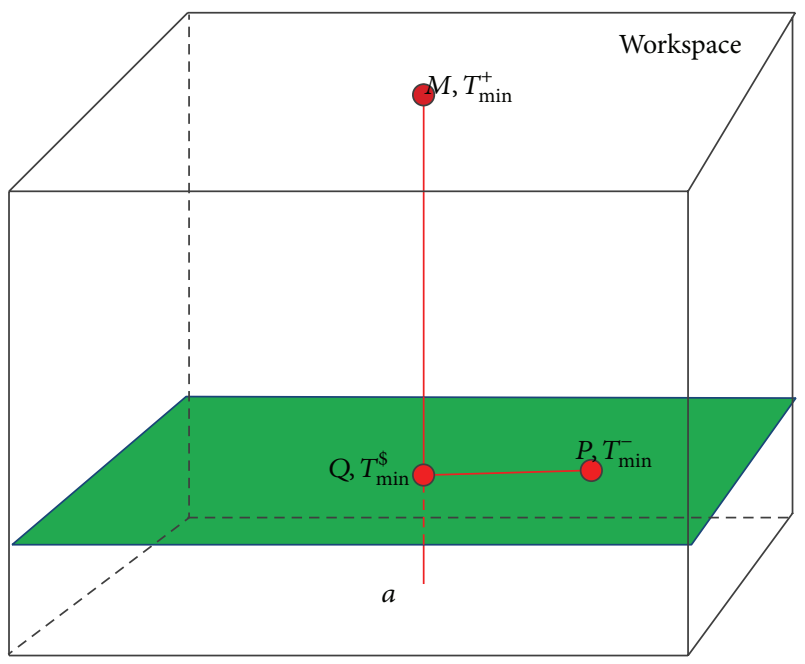

FIGURE 2: Schematic diagram of the three performance indices.

where $T_{\min }^{+}$is the minimum cable tension at the position $M$; $T_{\min }^{-}$is the minimum cable tension at any specified position $P ; T_{\min }^{\$}$ is the minimum cable tension at the position $Q$.

Referring to Figure 2, the definitions of the three performance indices reflect the relative differences in the minimum cable tensions between the specified position and the target position. As a matter of fact, the positions $M$ and $Q$ possess the maximum $T_{\min }$ on the vertical midline and on the horizontal plane highlighted with green, respectively. Therefore, the minimum cable tension at the specified position is smaller than the one at the target position, so the values of the three performance indices are always within the interval $(0,1]$. In detail, $\mathfrak{R}_{\perp}$ indicates the relative difference in the minimum cable tensions between the specified position $Q$ and the target position $M ; \Re_{-}$means the relative difference in the minimum cable tensions between the specified position $P$ and the target position $Q$; and furthermore, $\mathfrak{R}$ shows the relative difference in the minimum cable tensions between the specified position $P$ and the target position $M$.

3.3. SMCTW Generation Algorithm. Furthermore, SMCTW is composed of the positions that have specified minimum cable tensions. According to [10], the stiffness that can improve stability of a cable-based robot increases as the cable tensions do. Therefore, the minimum cable tensions can be employed to evaluate the stability of a cable-based robot. The positions in which the minimum cable tensions exceed a specified value are considered to the stable ones.

The workflow of SMCTW generation algorithm can be summarized as follows.

(1) Input the real-time platform position $\mathbf{X}_{i}$ in the workspace $(i=1,2, \ldots, N . N$ is the total number of positions) and the minimum cable tension limit $T_{\min }^{*}$; the external wrench $\mathbf{F}_{\mathbf{e}}\left(\mathbf{X}_{i}\right)$; the gravity $\mathbf{W}_{g}\left(\mathbf{X}_{i}\right)$ of the robot platform.

(2) Calculate the structure matrix $\left(\mathbf{X}_{i}\right)$.

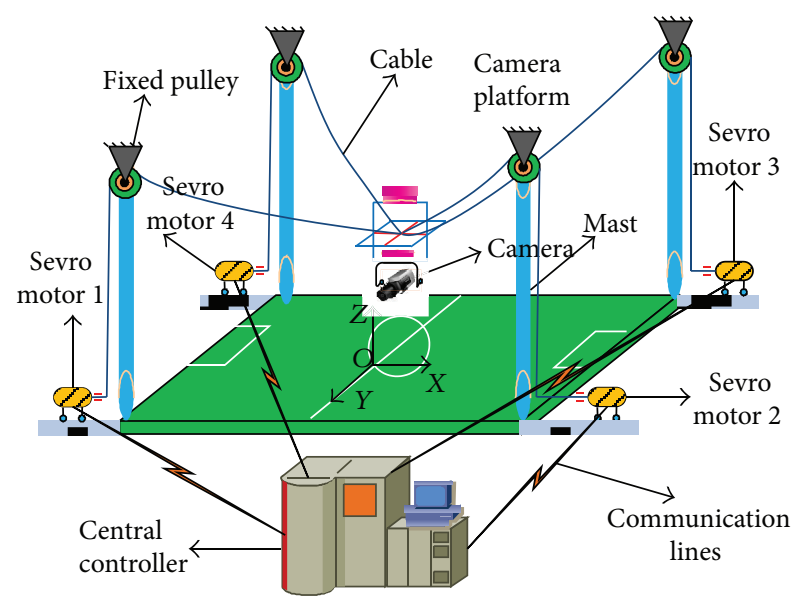

FIGURE 3: Schematic diagram of a camera robot.

(3) Obtain the null space matrix $\operatorname{Null}\left(\mathbf{W}\left(\mathbf{X}_{i}\right)\right)$ of the structure matric $\mathbf{W}\left(\mathbf{X}_{i}\right)$.

(4) Find the optimum value $T^{*}\left(\mathbf{X}_{i}\right)=\mathbf{W}\left(\mathbf{X}_{i}\right)^{+}\left(-\mathbf{F}_{e}-\right.$ $\left.\mathbf{W}_{g}\right)+\operatorname{Null}\left(\mathbf{W}\left(\mathbf{X}_{i}\right)\right) \lambda_{i}^{*}$ using convex programming theory and (10).

(5) Calculate the minimum cable tension $T_{\min }\left(\mathbf{X}_{i}\right)$ using (11).

(6) Judge whether $T_{\min }\left(\mathbf{X}_{i}\right)$ exceeds $T_{\min }^{*}$. If it does, record and output $\mathbf{X}_{i}$; if not, go to the next position.

(7) Judge if $\mathbf{X}_{i}$ is the last position. If not, go to (1) and solve $\mathbf{T}^{*}\left(\mathbf{X}_{i+1}\right)$ and $T_{\min }\left(\mathbf{X}_{i+1}\right)$ for the next position $\mathbf{X}_{i+1}$; if it is, record and output the position $\mathbf{X}_{i}$ and stop the calculation.

\section{Examples}

An example is now presented in order to illustrate the distributions of the minimum cable tensions in the workspace and the relationship between the three performance indices and the stability. A spatial three-degree-of-freedom cablebased parallel robot with four cables that is called camera robot is used for simulation and analysis [11]. It is worth noting that the platform is assimilated to a unique point mass.

4.1. Description of the Camera Robot. The camera robot structure is displayed in Figure 3, consisting of a moving camera platform with 3 DOF connected to the fixed pulleys by four cables. And the mass of the camera platform $m_{c}=10 \mathrm{~kg}, \mathbf{T}_{\max }^{s}=\left[\begin{array}{llll}300 & 300 & 300 & 300\end{array}\right]^{T} \mathrm{~N}$, and $\mathbf{T}_{\min }^{s}=$ $\left[\begin{array}{llll}10 & 10 & 10 & 10\end{array}\right]^{T} \mathrm{~N}$. In the example shown here, the origin of the fixed base reference frame $O X Y Z$ is located at the bottom of mastl. The position vectors in which the cables enter the pulleys are $\mathbf{A}_{\mathbf{1}}=\left[\begin{array}{lll}0 & 0 & 23\end{array}\right]^{T} \mathrm{~m}, \mathbf{A}_{\mathbf{2}}=\left[\begin{array}{lll}37 & 0 & 23\end{array}\right]^{T} \mathrm{~m}$, $\mathbf{A}_{\mathbf{3}}=\left[\begin{array}{lll}37 & 40 & 23\end{array}\right]^{T} \mathrm{~m}$, and $\mathbf{A}_{\mathbf{4}}=\left[\begin{array}{lll}0 & 40 & 23\end{array}\right]^{T} \mathrm{~m}$. Therefore, the midpoint of the horizontal surface is at $x=18.5 \mathrm{~m}$ and $y=20 \mathrm{~m}$. 


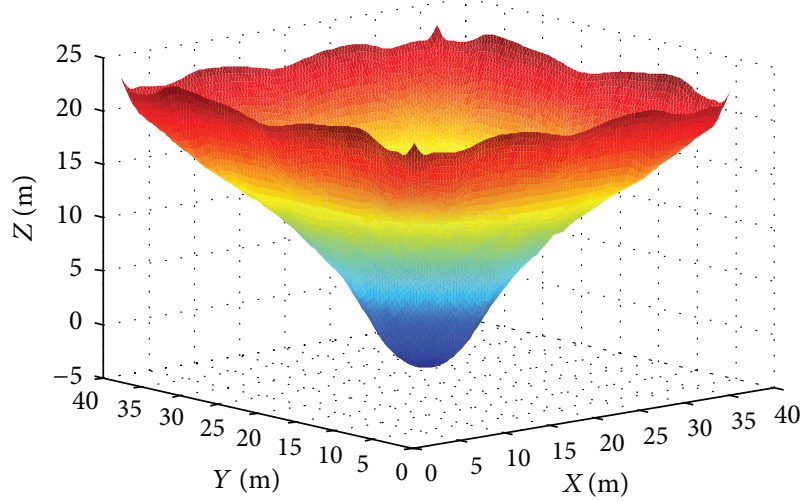

Figure $4: T_{\min }=30 \mathrm{~N}$ in the workspace.

4.2. Results and Discussion. As shown in Figure 4, the surface on which the minimum cable tensions equal $30 \mathrm{~N}$ is obtained in the whole workspace. Of course, it is worth noting that the positions within the surface retain the minimum cable tensions that are bigger than $30 \mathrm{~N}$. Its colors are worthy of note that represent the elevation along $Z$-direction. Moreover, one of its features is the symmetry about $X$-direction and $Y$ direction just because the horizontal sections are rectangles.

The minimum cable tensions on the different vertical and horizontal planes are depicted in Figure 5. One may observe that the upper and center positions possess bigger $T_{\min }$ than the ones around them on the vertical middle planes from Figures 5(a) and 5(b). It is evident that the curves having the same $T_{\min }$ are at the same elevation because they are the front and lateral views of SMCTW, respectively. Even more important, the interior curves hold bigger $T_{\min }$ than the ones outside. It is worth noting that the shape of the curve in Figure 5(c) provides a detailed statement about the change of $T_{\min }$ on the central axis of the vertical plane. And in Figure 5(d), as it can be observed that the contours of $T_{\min }$ are a family of similar concentric ellipses, which is because the sections of the horizontal plane are rectangles. Meanwhile, a brief view of the contours indicates that the curves inside possess bigger $T_{\min }$ than the ones outside because of the uniformity tensions in all cables at the positions next to the center. Moreover, the contours spread as $z$ increases because the upper workspace is bigger than the lower one. Altogether, the different profiles are displayed in Figure 5. As expected, further insight into the integral shape of SMCTW can be obtained from them. From above it can be concluded that the combination of all the curves in Figure 5 is in conformity with Figure 4 that displays $T_{\min }=30 \mathrm{~N}$ in the workspace.

The minimum cable tensions on two different vertical planes are depicted in Figure 6. Together with Figure 5(b), one may observe that the minimum cable tensions vary with different $x$. Note that both of the contours are closed on the middle vertical plane in Figure 5(b) while some of them are not closed in Figure 6(a). Furthermore, one evident feature of Figure 6(b) is that the minimum cable tensions are not always continuous, and this is because only the positions near the pulleys meet the minimum cable tension requirement at the upper boundary of the workspace, while the same discontinuity occurs at the same time in Figure 6(a) at $T_{\min }=$ $20 \mathrm{~N}$. The reason for this is that the minimum cable tension at the bottom of the vertical midline is bigger than $30 \mathrm{~N}$.

The minimum cable tensions at different elevations are depicted in Figure 7. Together with Figure 5(d), one may observe that the curves of minimum cable tensions vary with different $z$. Note that the curves that hold bigger $T_{\min }$ are surrounded by the ones that do not; this is because the positions next to the center have relatively great uniformity of the cable tensions. It is concluded that the platform has better stability at the interior positions.

The three performance indices above are computed using (12) in this section for the camera robot. And the results are displayed in Figure 8. As expected, the performance index $\mathfrak{R}_{\perp}$ increases as $z$ increases along the vertical midline shown in Figure 8(a). It is worth noting that the shape of the curve that is a nonlinear function of the elevations is similar to the shape of the curve in Figure 5(c), this is because the curve shown in Figure 8(a) is obtained by the operation, namely, $T_{\min } / T_{\min }^{\max }\left(T_{\min }\right.$ is the minimum cable tension at every vertical midline elevation and $T_{\min }^{\max }$ is the maximum tension among them on the vertical midline). It can be seen from Figure 8(b) that the positions that hold the bigger $T_{\text {min }}$ are the ones that are close to the center of every horizontal plane. That is to say, the upper and central positions possess bigger $T_{\min }$ than others, thereby improving the stiffness and stability of the end-effector, and further, the platform can move more smoothly. In addition, the performance index $\Re$ is depicted from Figures $8(\mathrm{c})$ to $8(\mathrm{f})$. With Figures $8(\mathrm{c})$ and $8(\mathrm{~d})$ together, it is evident that the curves that have the same $\mathfrak{R}$ are at the same elevation along $Z$-direction because they are front and lateral views of $\mathfrak{R}$ in the workspace, respectively. A further view of the inverted triangular figure curves indicates that the interior positions retain bigger $\mathfrak{R}$ than others because of the uniformity of the cable tensions. While the surface that $\mathfrak{R}$ forms on the horizontal plane is displayed in Figure 8(e), it was obvious that the surface is similar to the one in Figure 8(b); this is because $\Re$ is $\Re_{\perp 10}$ (highlighted in Figure 8(a)) times as big as $\Re_{-}$at $z=10 \mathrm{~m}$. Completely, a specified value of $\Re$ is described in the last of Figure 8, and note that it is similar to Figure 4 because of the meaning of $\Re$. Indeed, the meaning is the proportion of the maximum tension in the whole workspace. It should be pointed that the positions within the surface meet the specified stability requirement. Referring to Figure 8 , it turns out that an increase in the performance index $\mathfrak{R}_{\perp}$ leads to a corresponding increase in elevation along $Z$-direction, while an increase in the performance index $\mathfrak{R}_{\text {- }}$ leads to a corresponding position closer to the center of the horizontal plane, therefore increasing the minimum cable tension at the specified positions. The following facts are required to be apprehended: (1) it is well known that the stability is defined in general terms as "the likelihood that an external disturbance will disturb the end-effector from a given equilibrium position" [12]. It is evident that the end-effector will be most likely to be disturbed while the external disturbance is along the weakest constraint direction. Therefore, the stability of the end-effector means that the end-effector cannot depart 


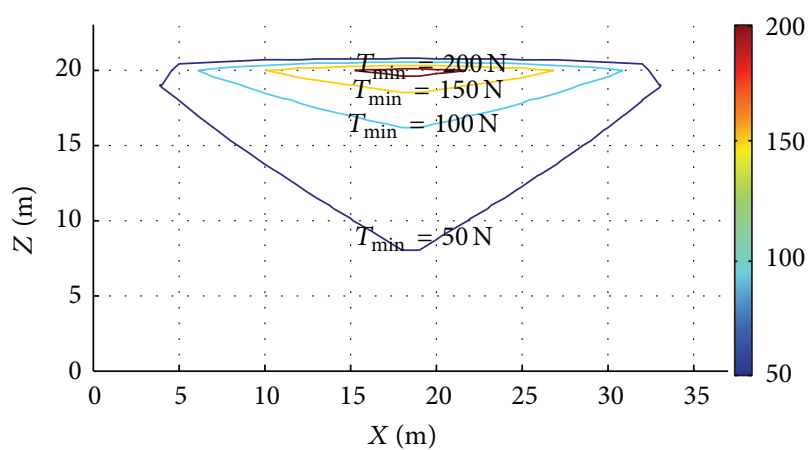

(a) $T_{\min }$ on the middle vertical plane $y=20 \mathrm{~m}$

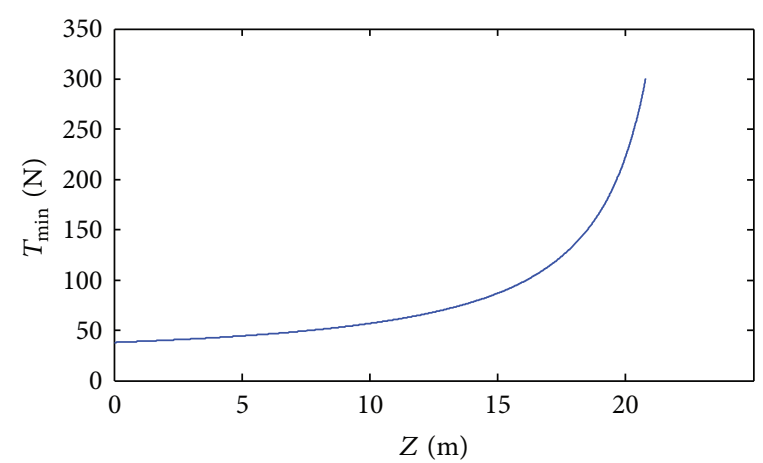

(c) $T_{\min }$ on the vertical midline $x=18.5 \mathrm{~m}$ and $y=20 \mathrm{~m}$

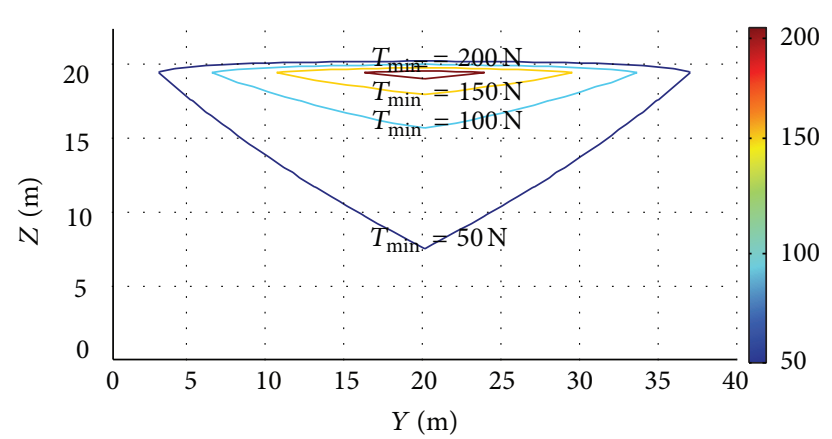

(b) $T_{\min }$ on the middle vertical plane $x=18.5 \mathrm{~m}$

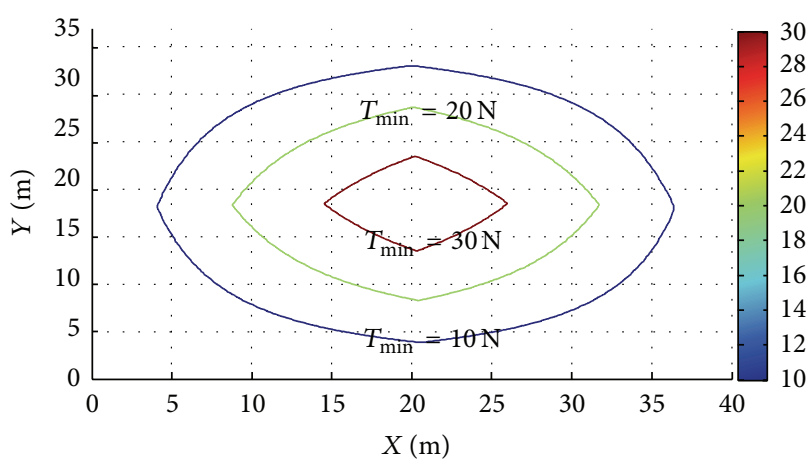

(d) $T_{\min }$ on the horizontal plane $z=0 \mathrm{~m}$

FIGURE 5: $T_{\min }$ on the sections of the workspace for the camera robot.

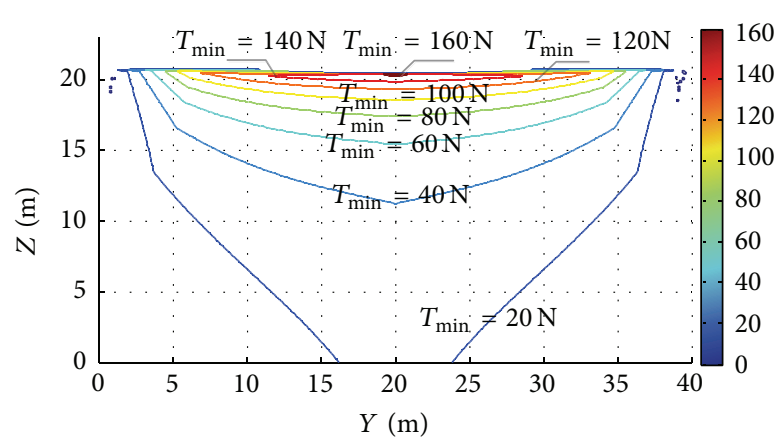

(a) $x=10 \mathrm{~m}$

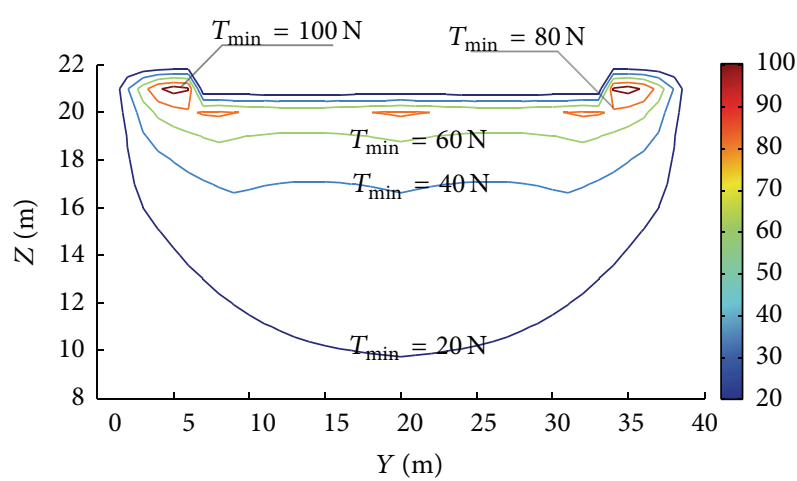

(b) $x=5 \mathrm{~m}$

FIGURE 6: $T_{\min }$ on the vertical planes paralleling with the plane YOZ.

from the present position while an external disturbance exerting along the weakest constraint direction. (2) Apart from the abovementioned, it is important to notice that the direction of the minimum cable tension is the weakest constraint direction in all the ones at the present position. As it is seen from the facts above that as the performance indices increase, so do the minimum cable tensions and the constraints of the weakest direction, further improving the stability of the end-effector. In other words, the stability of the end-effector is determined partially by the performance indices including the minimum cable tensions. Furthermore, it turns out that an increase in the three performances indices leads to a corresponding increase in the stability of the end-effector.

From the simulation results and analysis above it can be concluded that the camera robot can move stably and reliably at the positions that contain bigger performance indices just due to the relatively large minimum cable tensions and uniformity of the tensions.

\section{Conclusions}

The minimum cable tension distributions for completely restrained cable-based parallel robots are discussed using 


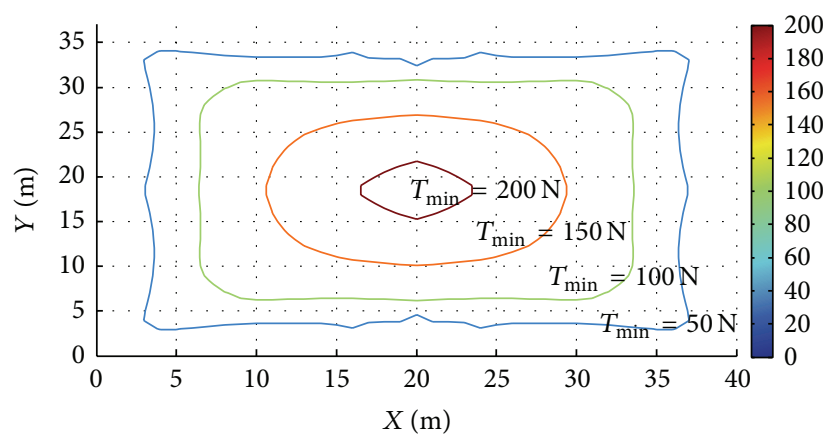

(a) $T_{\min }$ on the horizontal plane $z=20 \mathrm{~m}$

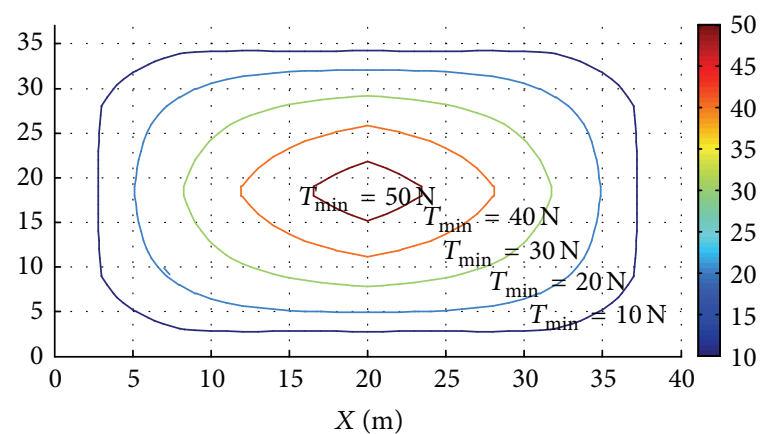

(b) $T_{\min }$ on the horizontal plane $z=10 \mathrm{~m}$

FIgURE 7: $T_{\min }$ on the horizontal planes paralleling with the plane XOY.

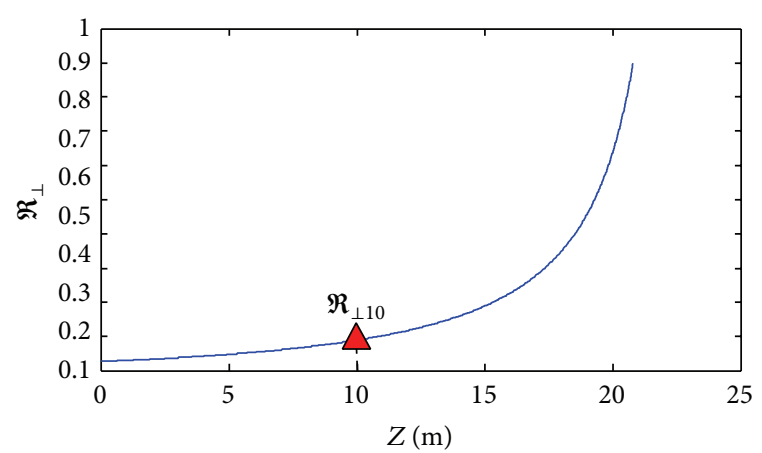

(a) $\Re_{\perp}$ on the vertical midline $x=18.5 \mathrm{~m}$ and $y=20 \mathrm{~m}$

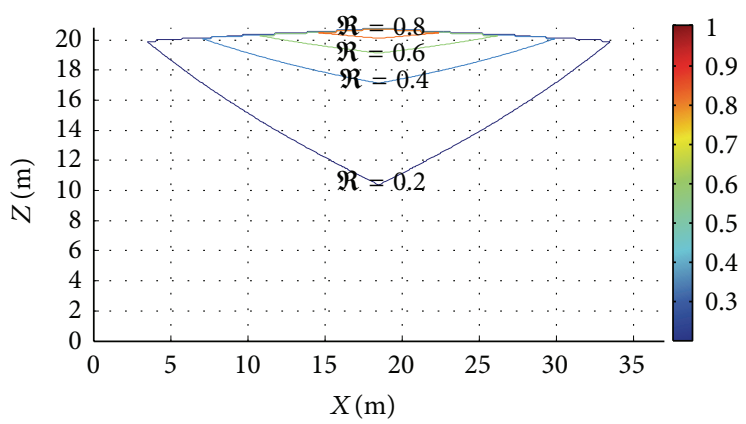

(c) $\Re$ on the middle vertical plane $y=20 \mathrm{~m}$

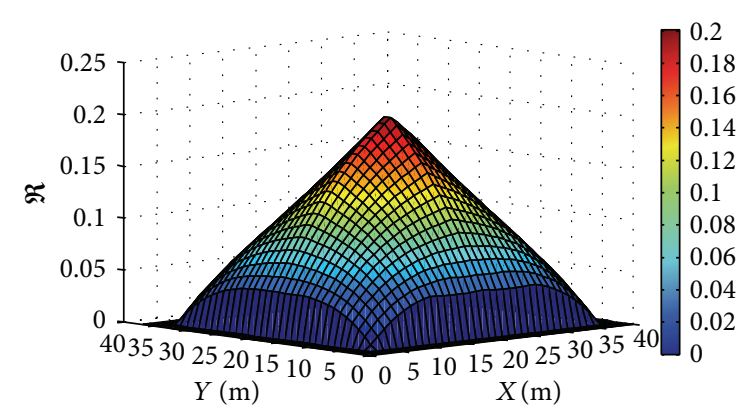

(e) $\mathfrak{R}$ on the horizontal plane $z=10 \mathrm{~m}$

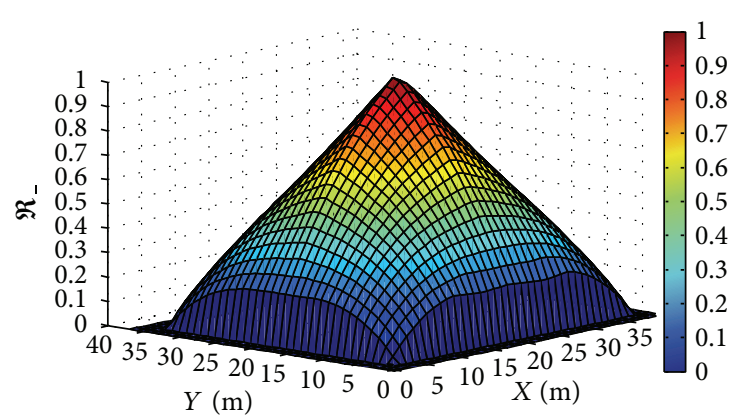

(b) $\Re_{-}$on the horizontal plane $z=10 \mathrm{~m}$

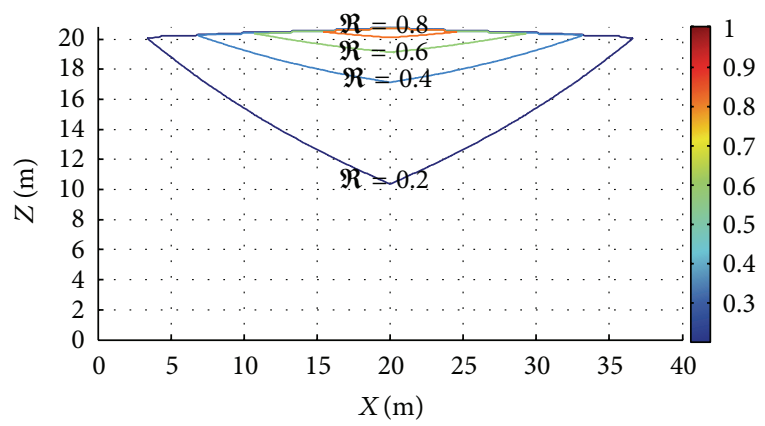

(d) $\Re$ on the middle vertical plane $x=18.5 \mathrm{~m}$

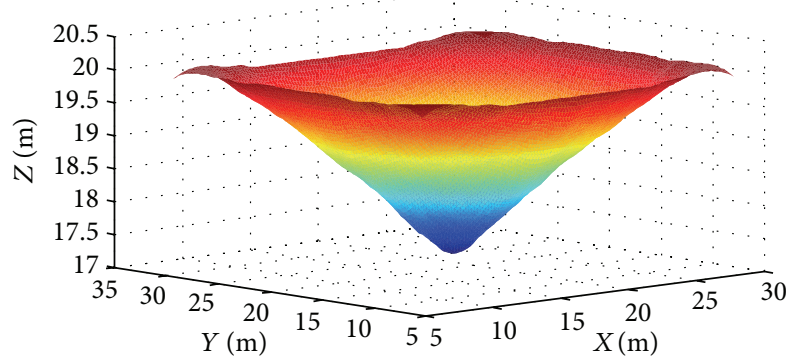

(f) $\mathfrak{R}=0.4$ in the workspace

FIGURE 8: The three force performance indices in the workspace. 
the three performance indices based on the cable tension determining. Solutions to the problems of minimum cable tension distributions are presented to a typical camera robot. Simulation results of the three performance indices show that as the three performance indices increase, so do the minimum cable tensions and the constraints of the weakest direction, therefore improving the stability of the movement. In the whole workspace, the positions in the center and on top of the workspace possess bigger minimum cable tensions than others, leading to having better stability. It is important to notice that the positions within the surface formed by a specified $\mathfrak{R}$ have better stability. Furthermore, this research can potentially be extended to deal with the incompletely restrained and redundantly restrained cable-based parallel robots.

\section{Conflict of Interests}

The authors declare that there is no conflict of interests regarding the publication of this paper.

\section{Acknowledgment}

The authors gratefully acknowledge the financial support of National Science Foundation of China under Grant nos. 51175397 and 51105290.

\section{References}

[1] J. P. Merlet, "Wire-driven parallel robot: open issues," in Romansy 19-Robot Design, Dynamics and Control, pp. 3-10, CISM International Centre for Mechanical Sciences, Springer, Vienna, Austria, 2013.

[2] Y. Mao and S. K. Agrawal, "Design of a cable-driven arm exoskeleton (CAREX) for neural rehabilitation," IEEE Transactions on Robotics, vol. 28, no. 4, pp. 922-931, 2012.

[3] P. Lafourcade, L. Michellm, and R. Claude, "Design of a parallel wire-driven manipulator for wind tunnels," in Proceedings of the Workshop on Fundamental Issues and Future Research Directions for Parallel Mechanisms and Manipulators, pp. 187-194, Quebec, Canada, 2002.

[4] B. Y. Duan, Y. Y. Qiu, F. S. Zhang, and B. Zi, "On design and experiment of the feed cable-suspended structure for super antenna," Mechatronics, vol. 19, no. 4, pp. 503-509, 2009.

[5] R. Verhoeven, Analysis of the workspace of tendon-based Stewart platform [M.S. thesis], University of Duisburg-Essen, Duisburg, Germany, 2004.

[6] C. Gosselin and M. Grenier, "On the determination of the force distribution in overconstrained cable-driven parallel mechanisms," Meccanica, vol. 46, no. 1, pp. 3-15, 2011.

[7] M. Hassan and A. Khajepour, "Optimization of actuator forces in cable-based parallel manipulators using convex analysis," IEEE Transactions on Robotics, vol. 24, no. 3, pp. 736-740, 2008.

[8] L. Mikelsons, T. Bruckmann, M. Hiller, and D. Schramm, "A real-time capable force calculation algorithm for redundant tendon-based parallel manipulators," in Proceeding of the IEEE International Conference on Robotics and Automation (ICRA '08), pp. 3869-3874, Pasadena, Calif, USA, May 2008.
[9] P. H. Borgstrom, B. L. Jordan, G. S. Sukhatme, M. A. Batalin, and W. J. Kaiser, "Rapid computation of optimally safe tension distributions for parallel cable-driven robots," IEEE Transactions on Robotics, vol. 25, no. 6, pp. 1271-1281, 2009.

[10] S. Behzadipour and A. Khajepour, "Stiffness of cable-based parallel manipulators with application to stability analysis," Journal of Mechanical Design, vol. 128, no. 1, pp. 303-310, 2006.

[11] Y. Su, Y. Qiu, and P. Liu, "Optimal cable tension distribution of the high-speed redundant driven camera robots considering cable sag and inertia effects," Advances in Mechanical Engineering, vol. 2014, Article ID 729020, 11 pages, 2014.

[12] P. M. Bosscher, Disturbance robustness measures and WrenchFeasibile workspace generation techniques for cable-driven robots [Ph.D. thesis], 2004. 


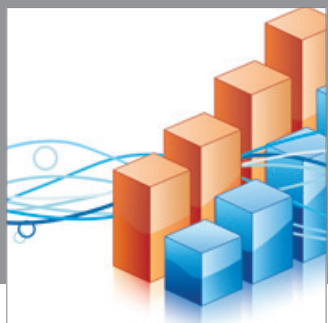

Advances in

Operations Research

mansans

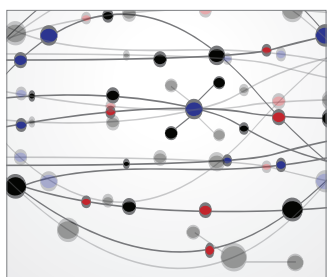

The Scientific World Journal
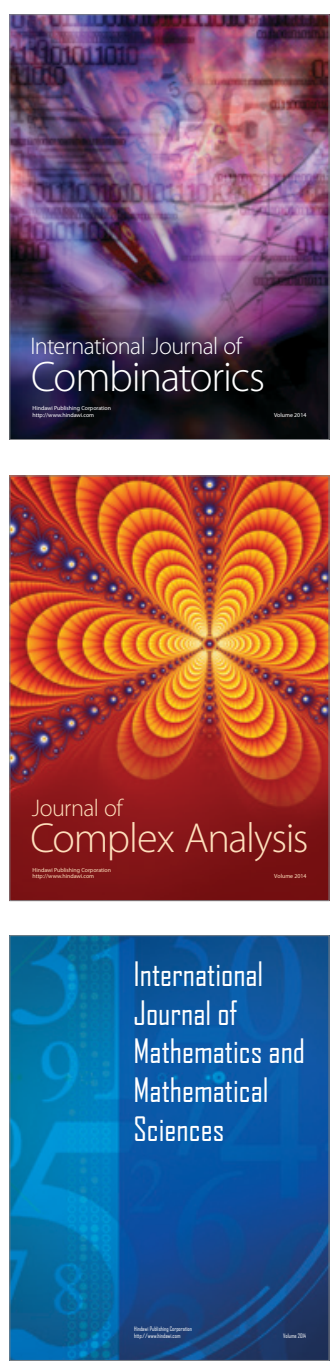
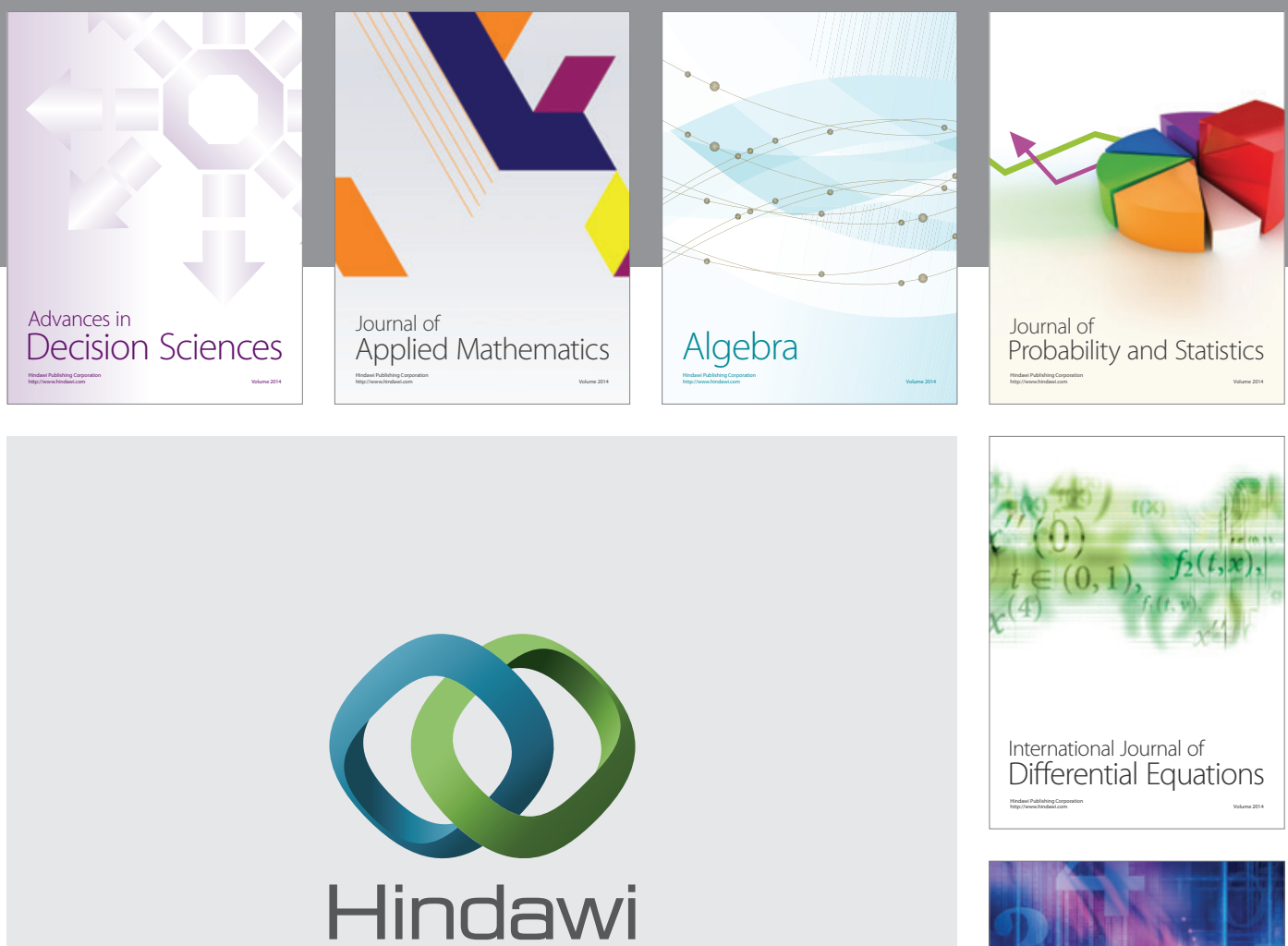

Submit your manuscripts at http://www.hindawi.com
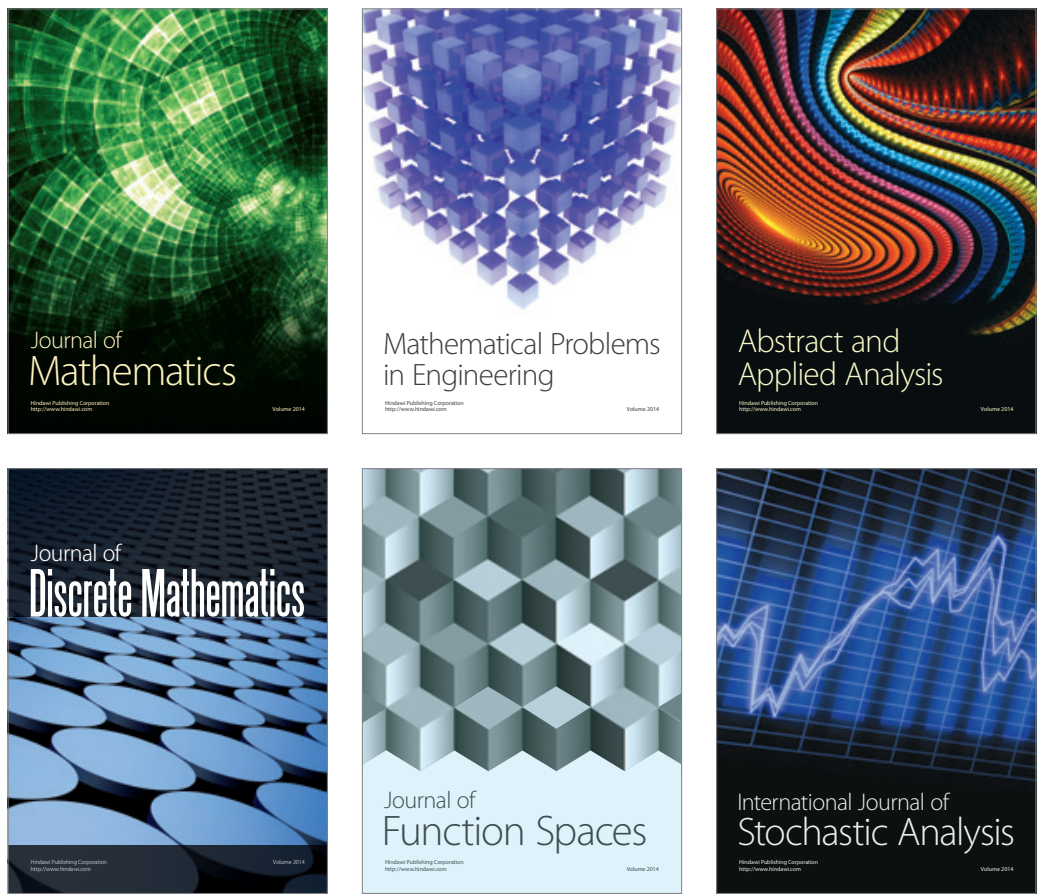

Journal of

Function Spaces

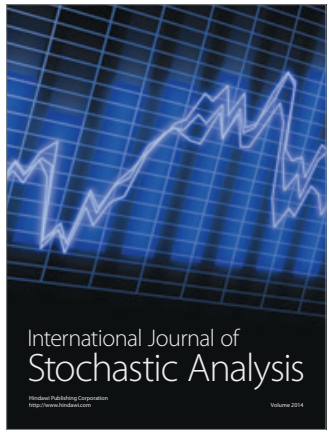

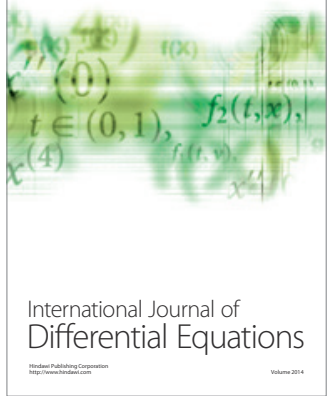
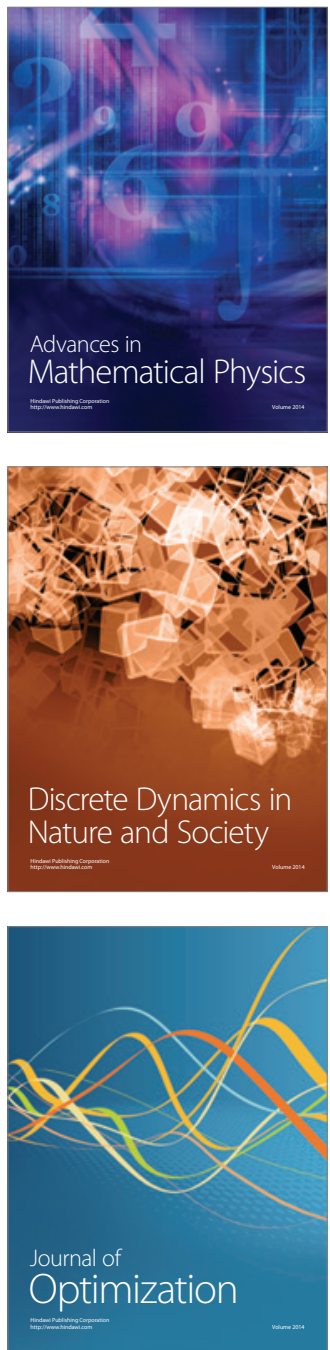